Original Research Paper

\title{
Predicting the Remaining Lifetime of Distribution Transformers using Machine Learning
}

\author{
${ }^{1}$ Justice Aning, ${ }^{2}$ Puguo Gbene George and ${ }^{2}$ Ntiminity Abontakoyah Enoch \\ ${ }^{1}$ Department of Computer Science, Sunyani Technical University, Sunyani, Ghana \\ ${ }^{2}$ Department of Electrical \& Electronic Engineering, Sunyani Technical University, Sunyani, Ghana
}

\author{
Article history \\ Received: $12-08-2020$ \\ Revised: 16-10-2020 \\ Accepted: 28-10-2020 \\ Corresponding Author: \\ Justice Aning \\ Department of Computer \\ Science, Sunyani Technical \\ University, Sunyani, Ghana \\ Email: aning421@gmail.com
}

\begin{abstract}
Distribution Transformer is a crucial element in deciding the power flow in large power systems. Their better performance implies high power system efficiency and enhanced power transfer capability. However, various Distribution Transformer failures in the recent past lead to power supply disturbance and have acquired much attention from the electrical intellectuals. It is of considerable significance to accurately get the running state of distribution transformers and timely detect the existence of potential transformer faults. This project work presents a predictive model to predict the potential of a distribution transformer failing before its expected years in service. Using Random Forest machine learning techniques, we examine transformer data from August 2010 to June 2019. Our experimental results reveal that a total of 90 distribution transformers were damaged within nine years. Thus, average the company losses ten (10) transformer in a year, which amount to the US $\$ 92300-95770$ per year. Also, most of the places that recorded rate of distribution transformer damage were a location that had mini and major factories around. Thus, the Sunyani Municipality recorded the highest transformer damage (12), representing 13\%, followed by Mim (10). Again, lighting strike was the significant causes of transformer damage. Thus twenty-one (21) out of the ninety (90) damage transformers was caused by a lightning strike. The results further show that $33.33 \%$ of the damage transformers were with $24.75-36.75 \%$ of their life expectancy. As low as $3.33 \%$ of the damage transformers have been in service for $73 \%$ of the life expectancy. From the study results, it can be concluded that a high percentage $(68.9 \%)$ of the damage transformers in the Bono, Bono East and Ahafo regions of Ghana have been in service less the half of its expected years of service. Rate-offaulty-occurrence, Type-of-faults-sustained and Tap-changer-type are the most significant factors that determine the number of years left for a distribution transformer to fail. We observed that the make of a transformer was of less importance in predicting the years left for a transformer to fail. Finally, the RMSE of 0.001639 and MAPE error of 0.001321 achieved by the proposed model shows that the proposed model fits very well to the dataset.
\end{abstract}

Keywords: Distribution Transformer, Machine Learning, Random Forest

\section{Introduction}

Transformers are considered as the most crucial and expensive piece of plant within a transmission and distribution networks system (Dewangan and Patel, 2017; Van Schijndel et al., 2006). Most transmission systems currently have vast populations of ageing transformers (Visser and Brihmohan, 2008), whiles loads on transformers keep increasing as the demand for electricity increases globally. Presently, economic strategies are calling for reduced maintenance as well as capital expenditure (Pickster, 2015; Rawal and Pandya, 2015). These challenges, which face utilities worldwide, necessitate improved management of transformers. Electrical transmission is an essential part of the Ghanaian energy industry. Currently, there are approximately 1,000 high-voltage power transmission transformers in service in Ghana. Unexpected failures of 
any of these transformers can cause substantial economic losses to supply authorities and consumers.

Besides, as one of the essential pieces of equipment in the power system, power and distribution transformers can directly influence the stability and safety of the entire power grid (Badune et al., 2013; Kimment and Matevosyan, 2018). If the transformer fails in operation, it will cause power to turn off and cause damage to the transformer itself and the power system, which may result in more considerable damage (Lin et al., 2018). Lifetime data analysis of power and distribution transformers are essential for a cost-efficient and risk minimized maintenance process (Badune et al., 2013).

The reliability of the electric grid is of paramount economic importance and with grid becoming smarter, we can effectively monitor the state of the power grid and its components. The goal is to make the expected performance quantifiable, make risks and costs predictable and controllable (Osorio and Sawant, 2003). Prediction of the remaining life of high-voltage distribution and power transformer is an essential issue for energy generators and distributors because of the need for planning maintenance and capital expenditures (Hong et al., 2009). Predicting the remaining life can be based on historic lifetime information about the transformer population (or fleet). Nevertheless, because the lifetimes of some transformers extend over several decades, transformer lifetime data are complicated (Hong et al., 2009).

Most transformer manufactures typically estimate the life span of transformers between 25 to 40 years. Although several transformers in service are approaching their expected age and others are over their expected age (Gorgan et al., 2012). Therefore, it is essential to estimate their remaining life to prevent any premature or sudden shutdown of transformers. However, to the best of author's knowledge, most existing studies in this field focused on developed countries, developing countries like Ghana have received little or no studies in this area. On the other hand, environmental factors which are proven to affect failures of transformers differ from country to county. This makes it challenging to generalized the study outcome from one country to another with different environmental factors. Hence this study aims to apply machine-learning techniques to determine the factors that contribute to the failure of distribution transformers in service in three regions of Ghana. Also, predict the remaining life of distribution transformer in use based on the identified factors. Specifically, we seek to:

a) Identify the causes of failures in the distribution transformer in the Sunyani Municipality

b) Measure the degree of association between the factors in (a) and failure of a distribution transformer and obtain key significate factors c) Predict the remaining lifetime of distribution transformers currently in service using identified critical factors in (b)

The following research questions are set to guide the current study:

1. What factors lead to failures of distribution transformers?

2. What is the degree of association between these factors and distribution transformer failure?

3. How possible is it to predict the probability of failure of the transformers currently in service?

4. The remaining sections of this study are organized as follows: Section 2 presents the methods and techniques adopted for the study. In section 3, we present study results and discussions. Finally, we round up in section 3 with study conclusions, recommendations and future works

\section{Literature Review}

\section{Machine Learning Algorithms}

"Machine Learning is the science of getting computers to learn and act as humans do and improve their learning over time in an autonomous fashion, by feeding them data and information in the form of observations and real-world interactions" (Faggella, 2018). Machine learning have been applied in several sectors and have achieved good results. For example financial market (Nti et al., 2019a; 2020a-c), education (Adejo and Connolly, 2018; Nti and Quarcoo, 2019; Tran et al., 2017), energy (Huber et al., 2018; Nti et al., 2020d-e), health (Futoma et al., 2020; Ngiam and Khor, 2019; Wiens et al., 2019), agriculture (Airlangga and Liu, 2019) and more. There are numerous machine-learning algorithms, however, we present two of these algorithms that are normally used in this field.

\section{Support Vector Machine (SVM)}

SVM recently has become an essential machine learning tool for tasks, including regression (Nti et al., 2020d) and classification. SVM is supervised machine learning algorithm that serves as the inline separator inserted between 2 data nodes to detect 2 different classes in the multidimensional environs, which can be employed for both regression and classification problems (Nti et al., 2020d; 2019b).

\section{Decision Tree (DT)}

A decision tree is a flow-chart-like tree structure that uses a branching technique to clarify every single likely result of a decision. Every discrete node within the tree embodies a test on a precise variable and each branch is the outcome of that test (Nti et al., 2020d; 2019a). The 
interpretability and simplicity of DT and their low-slung computational cost and the ability to represent them graphically has contributed to the increase in their use for classification. A DT denotes a set of conditions or restrictions which are organized hierarchically and which are sequentially applied from a root to a terminal node or leaf of the tree (Nti et al., 2020d; 2019b).

\section{Related Work}

The important role play by transformer in the power industries has contributed to several studies aimed at estimating the life expectancy of transformers to prevent any unwanted failure. In this section we present few of this pertinent studies.

The evaluation and analysis of distribution transformer losses under non-linear load based on real data was carried out in (Said et al., 2010). The paper aimed at determine losses caused by harmonic and life expectancy of the distribution transformer. The study concluded that an increased in current harmonic distortion will result in a corresponding decrease in the expected life of the transformer. An update to the models used in estimating the remaining life of transformer paper insulation was presented in (Martin et al., 2014). An online algorithm to calculate and forecast transformer rating to assess the overload capability during short and long times was undertaken in (Alvarez et al., 2019). The possibility of developing a remaining life estimation and asset management decision model using diagnostics of transformer insulation characteristics was proposed in (Mharakurwa et al., 2019). The study adopted an integration of the fuzzy logic diagnostic tool and the fuzzy logic remnant life mapping model.

Despite the several techniques are available to estimating the ageing rate and loss of life of transformers, (Hosseinkhanloo et al., 2020) argues that utilities looking into the transformer fleet ageing need a better tool to improve the ageing rate of transformers.
Besides, as already stated in this study, the several factors that affect transformer ageing are environmental dependent which differs across regions. Hence making it a challenge to apply a model trained on data from a particular region to a new region.

\section{Materials and Methods}

This section presents a brief detail of the case study, sample size, the materials and methods adopted for the implementation of the proposed transformer failure prediction model using machine learning.

\section{Study Framework}

Figure 1 shows the framework for this project work and it illustrates the steps involved. There are three main phases involved in this framework, Data Collection and Integration, Data Transformation and Patterns Extraction. Before applying data mining algorithms on any data, it is essential to carry out some pre-processing tasks such as cleaning, integration, discretization and variable transformation (Berhanu and Abera, 2015).

\section{Data Collection}

We collected data on monthly load monitoring and failures of thirty-two (32) distribution transformers from August 2010 to June 2019 Table 1. Table 2 shows the features of our dataset. The features of our dataset were mixed with textual and numerical. Hence, we converted all textual features into numerical features. The tap changer type was converted to 0 for off-load and 1 for on-load, the state of the transformer, thus, in-service $=1$ and out of services $=0$. The different cause of damage was categorized as a vector $k=\{1,2,3,4\}$, where each cause was associated with a specific number. In all our dataset was a matrix of eleven columns and 1000 rows.

Table 1: Location of transformers

\begin{tabular}{|c|c|}
\hline \multicolumn{2}{|l|}{ Transformer locations } \\
\hline 1 Adabokrom & 17 Kofiko (Drobo) \\
\hline 2 Atomfuoso & 18 Kukuom \\
\hline 3 Ayamso (Mim) & 19 Kwabena Tenten \\
\hline 4 Bechem & $20 \mathrm{Mim}$ \\
\hline 5 Berekum & 21 Mim Sakyikrom \\
\hline 6 Biaso (Wanfie) & 22 Nkrankwanta \\
\hline 7 Dormaa & 23 Nsapor (Berekum) \\
\hline 8 Drobo & 24 Ntotroso/Kenyasi \\
\hline 9 Drobo Kofikrom & 25 Sampa \\
\hline 10 Duayaw Nkwanta & 26 Sampa Atamfoso \\
\hline 11 Gyasikrom & 27 Sampa Buko \\
\hline 12 Hwidiem & 28 Sampa (Small Water) \\
\hline 13 Jinjin Market & 29 Sankore (Kukuom) \\
\hline 14 Jinjin Market (Berekum) & 30 Sunyani \\
\hline 16 Kenyasi & 32 Wamfie \\
\hline
\end{tabular}




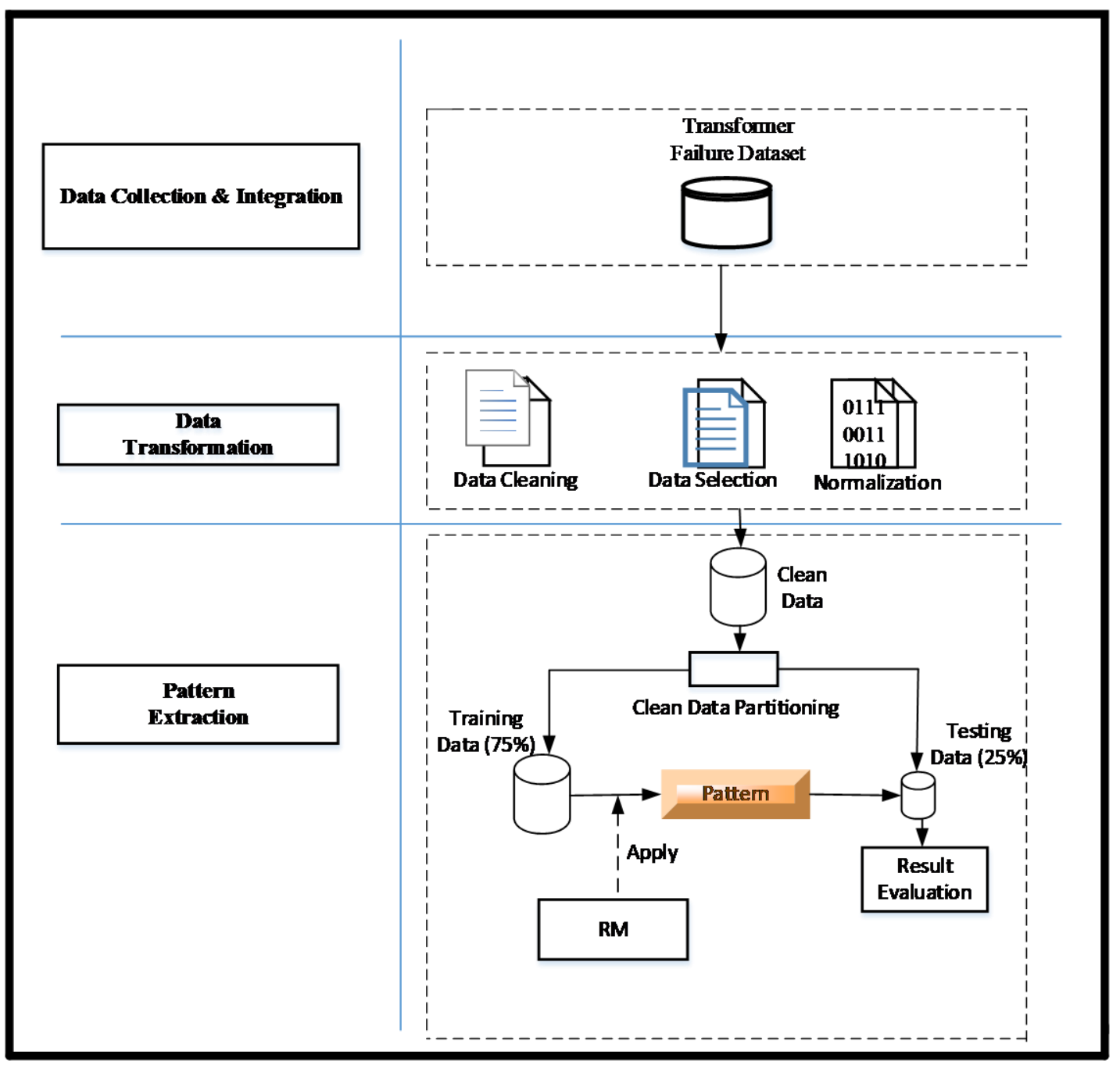

Fig. 1: Study framework

\section{Data Transformation}

We passed the collected Dataset (DS) through three distinct stages, (i) data cleaning which includes filling in missing values with mean values, smoothing noise, identification and removing of outliers where necessary and resolving data inconsistency. (ii) Data minimization and aggregation (iii) data reduction, where volumes of data were reduced but produce the same or similar analytical results using feature selection and feature extraction.

The clean data was partitioning into two, thus training dataset (Train_D) which accounted for $75 \%$ of the Dataset (DS) and the remaining 25\% was apportioned for testing (Test_D) the proposed model (Fig. 1). The training technique adopted for the current study was a supervised machine learning technique, where the intended input variables were entered into the network to produce the required output variable. The RF algorithm is applied to the Train_D and the model learning the pattern hidden in the dataset, we measure the accuracy and error metrics and determine if they within the accepted values. If they are, then the learnt pattern is then applied to the $($ Test_D) to make a prediction.

\section{The Adopted Machine Learning Algorithm}

Though several machine-learning algorithms can be employed for this study, however, the Random Forest (RF) algorithm was adopted based on the assessments in the following research work. In (Luo and Zhang, 2014; Nti et al., 2020d; 2019a-b; Vaghela et al., 2015), authors report that RF outperformed support vector machine SVM, AdaBoost and Artificial Neural Network (ANN). Again, the report of (Larivière and Van den Poel, 2005) shows that RF outperforms Linear Regression (LR). Furthermore, (Dai and Zhang, 2013; Di, 2014) reports that RF outperformed an enhance SVM. The superiority of RF in the machine-learning task is well known across different sectors. 
The RF is an ensemble-learning method that combines the performance of several decision tree algorithms to predict or classify the value of a variable (Nti et al., 2020d; 2019b). The RF can be used for both classification and regression machine learning task. In the RF approach, a large number of DTs are created, with each observation fed into every single decision tree. The maximum typical result for each observation is used as the final output. A different observation is served into all the trees and a majority vote is computed for apiece classification model. An error evaluation is made for the cases, which were not used throughout the tree building. This is known as Out-Of-Bag (OOB) error estimate, which is stated as a percentage. When an $R F$ receives an input of $(x)$, where $x$ is a vector made up of variable of different evidential features examined for a given training area, the RF builds several regression trees $(N)$ and averages the results. Therefore, that for $N$ tress $\left\{T(x)^{N}\right\}$ the FR regression predictor is given by Equation (1):

$$
f_{r f}^{N}(x)=\frac{1}{N} \sum_{N=1}^{N} T(x)
$$

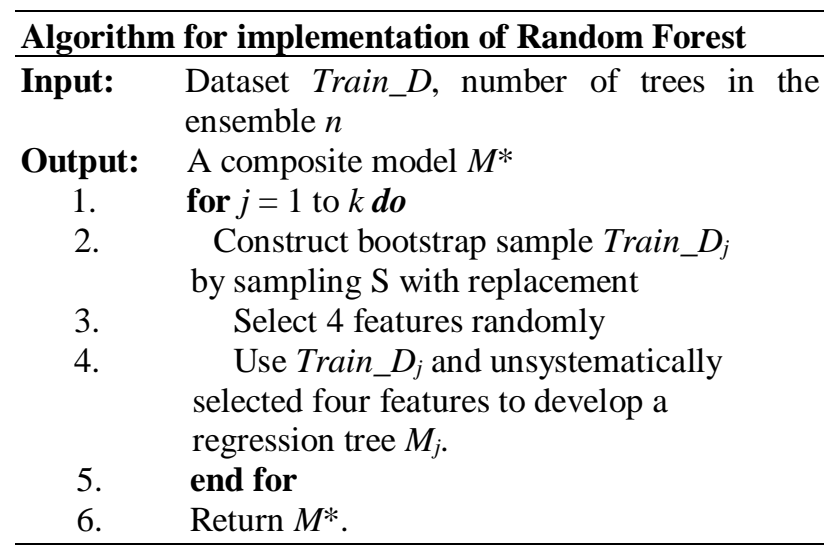

The Bootstrap Aggregating (Bagging) technique was used in assembling the decision trees for this study. Given a Train_D as given in Equation (2), the bagging technique generates a New_ $D_{i}$ new training dataset of size $N$ by sampling from the original training dataset Train_D with replacement. $N e w_{-} D_{i}$ is referred to as the bootstrap sample. By bootstrapping, some observations may be recurrent in each $N e w_{-} D_{i}$. This approach assist reduces variance and circumvents overfitting. Users specify the number of regression Trees $(T)$, in the current study, two hundred (160) trees were specified:

$\operatorname{Train}_{D}=\left\{\left(x_{1}, y_{1}\right),\left(x_{2}, y_{2}\right),\left(x_{3}, y_{3}\right), \ldots \ldots,\left(x_{N}, y_{N}\right)\right\}$

Choosing Variables to Split On: Grow unpruned regression tree with the following steps for each of the bootstrap samples: At the individual node, indiscriminately sample $K$ variables and select the most exceptional split among those variables $(K)$ rather than picking the most excellent split amid all predictors. This practice is sometimes called "feature bagging." We select a random subset of the predictors or features because the correlation of the trees in a standard bootstrap sample can be reduced. In this study $K=p / 3$.

The splitting principle, let assume that a partition is divided into $T$ constituencies $R_{1}, R_{2} \ldots, R_{T}$. We model the response as a constant $c_{k}$ in each constituency as proposed by (Wu et al., 2017) in Equation (3). The splitting principle at each node is to minimize the sum of squares. Hence, the best $\hat{c}_{t}$ is the average of $y_{i}$ in region $R_{t}$ as given in Equation (4):

$$
\begin{aligned}
& f^{(x)}=\sum_{t=1}^{T} c_{t} I\left(x \in R_{t}\right) \\
& c_{t} \operatorname{ave}\left(y_{i} \mid x_{i} \in R_{t}\right)
\end{aligned}
$$

Assume a splitting variable $j$ and split point $s$ and define the pair of half-planes:

$$
R_{1}(j, s)=\left\{X \mid X_{j} \leq s\right\} \text { and } R_{2}(j, s)=\{X \mid X \geq s\}
$$

Where the splitting variable $j$ and split point $s$ satisfy Equation (6):

$\left[\frac{\min }{c^{1}} \sum_{x_{i} \in R_{1(j, s)}}\left(y_{i}-c 1\right)^{2}+\frac{\min }{c^{2}} \sum_{x_{i} \in R_{2(j, s)}}\left(y_{i}-c 2\right)^{2}\right]$

When the best split is obtained, the dataset is partitioned into two resulting segments and echo the splitting procedure on each of the two segments. This splitting procedure is reiterated until a predefined ending criterion (threshold) is satisfied, five was set as the threshold for this study.

Table 2: Features of dataset

\begin{tabular}{ll}
\hline S/N & Feature \\
\hline 2. & Transformer Rating \\
3. & Make \\
& Tap changer Type (Off-load \\
4. & = O, On-load = 1) \\
5. & Voltage Level \\
6. & Output voltage \\
7. & Output Current \\
8. & \%Age as at failure \\
9. & State [0,1] \\
10. & Type of faults sustained be \\
11. & the transformer \\
\hline
\end{tabular}




\section{Evaluation Metrics}

In other to measure the performance of our proposed models, the following performance metrics defined in (Nti et al., 2020f-g; 2019b) were used to compare predicted values and actual values:

i. Root Mean Squared Error (RMSE): This index estimates the residual between the actual value and predicted value. A model has better performance if it has a smaller RMSE. An RMSE equal to zero represents a perfect fit

ii. Mean Absolute Percentage Error (MAPE): This index indicates an average of the absolute percentage errors, the lower the MAPE, the better

iii. The correlation coefficient $(\mathrm{R})$ : This criterion reveals the strength of relationships between actual values and predicted values. $\mathrm{R}$ ranges from 0 to 1 and a model with a higher $\mathrm{R}$ means better performance

iv. Accuracy

\section{Experimental Setup}

Eleven features Table 2 served as the input feature (independent variables) to predict the expected life in service $(y)$. The dataset is denoted as $(x, y)$, which consisted of pairs $\left(x_{i}, y_{i}\right)$ of features $\left(x_{i}\right)$ and remaining life $\left(y_{i}\right)$. The dataset was treated to (i) remove missing values by replacing them with average values where possible (ii) the dataset is of mixed type; thus, the features could be either continuous or categorical. Hence, we coded all categorical data to numerical values. (iii) scaling using the minimum-maximum function defined in Equation (7). (iv) we calculated the expected life of the transformers using Equation (8). $E_{\text {years }}=$ the expected years of a transformer in service before failure and $\left(Y_{\text {service }}\right)=$ is the actual years' a transformer spends in service before the damage. (v) dimensionality reduction using feature selection to obtained better learning performance:

$x^{\prime}=\frac{x-x_{\max }}{x_{\max }-x_{\min }}$

Remaining yearsbeforedamage $(y)=E_{\text {years }}-Y_{\text {service }}$

The RF model in this study was implemented through the random Forest package in Weka 3. The hyperparameters setting include the number of trees (ntree) and the total variables randomly sampled as candidates at each split (mtry). We optimized these hyperparameters using a ten (10) fold CrossValidation (CV) process searching the best parameters from a predefined grid of parameters ntree $=[15-160]$ and mtry $=[1: 8]$. Best results were obtained with ntree $=160$ and $m$ try $=2$.

\section{Results and Discussion}

This section presents the experimental setup, the results and discussion of the proposed RF prediction framework for predicting transformer failure. We present the results in table, frequency and charts.

\section{Descriptive Statistics}

\section{Failure (Damaged) Rate}

Table 3 shows the failure (Damage) rate of transformers in all substation under study. We observed that from the period of August 2010 to June 2019, there had been a total of ninety (90) distribution transformer that have damaged. The results show that Sunyani Municipality recorded the highest transformer damage (12), representing 13\%, followed by Mim (10), representing $11 \%$ and Tepa (8) representing 9\%. We further tried to estimate the damage cost of the 90 transformers using US \$9230-9577 (Alibaba.com, 2018) as the average cost. It was revealed that the company has lost within \$830,700.00-861,930.00 from August 2010 to June 2019.

\section{Causes of Damage}

The causes of the damage were analyzed and Fig. 2 shows the outcome. We observed that a lightning strike caused twenty-one (21) out of the ninety (90) damage transformers, the cause of damage for thirtysix (36) was not known (i.e., nineteen (19) unknown and 17 not immediately known). The great causes of damage due to lighting confirms the report of (Dewangan and Patel, 2017) that lighting strikes contribute to the highest cause of distributionstransformer damage. It was further observed that Mim recorded $23.8 \%$ out of the twenty-one (21) transformer damaged by lightening. Out of the seven transformers that were damaged by overheating Sunyani recorded $42.9 \%$. Thus, most of the transformers damaged in the Sunyani Municipality were due to overheating.

The usage life of transformers before damage was analyzed (Fig. 3). The results show that $33.33 \%$ of the damage transformers were with $24.75-36.75 \%$ of their life expectancy. As low as $3.33 \%$ of the damage transformers have been in service for $73 \%$ of the life expectancy. From the results, it can be concluded that a high percentage $(68.9 \%)$ of the damage transformers in the Bono, Bono East and Ahafo regions of Ghana have been in service less the half of its expected years of service. 


\section{Model Training and Testing}

Figure 4 shows the Out-of-bag error rate of the proposed random forest classifier. The aim here was to obtain the right number of estimators that will offer less error. The minimum number of estimators was set to fifteen (15) and the maximum to two hundred (200). The result shows that as the number of estimators increasing the error, the error margin reduces. However, the error increased when the number of estimators increased above one hundred and seventy-five (175). Hence, for this study, the number of estimators was set to one hundred and sixty (160).

\section{Feature Importance Ranking}

The correlation between each input feature and the expected output (Years to fail) was tested. Figure 5 shows the features importance ranking. The result shows that the Rate-of-faulty-occurrence, Type-of-faults-sustained and Tap-changer-type are the most significant factors that determine the number of years left for a distribution transformer to fail. We observed that the make of the transformer is of less importance in predicting the years left for a transformer to fail. Furthermore, we observed that moisture ingress if not attended as early as possible causes a high rate of damage in a transformer.

Table 3: Failure rate analysis

\begin{tabular}{|c|c|c|}
\hline Location & No. Failures (F) & Percentage $(\%)$ \\
\hline Adabokrom & 1 & 1 \\
\hline Atomfuoso & 1 & 1 \\
\hline Ayamso (Mim) & 1 & 1 \\
\hline Bechem & 4 & 4 \\
\hline Berekum & 6 & 7 \\
\hline Biaso (Wanfie) & 1 & 1 \\
\hline Dormaa & 4 & 4 \\
\hline Drobo & 5 & 6 \\
\hline Drobo Kofikrom & 1 & 1 \\
\hline Duayaw Nkwanta & 6 & 7 \\
\hline Gyasikrom & 1 & 1 \\
\hline Hwidiem & 6 & 7 \\
\hline Jinjin Market & 1 & 1 \\
\hline Jinjin Market (Berekum) & 1 & 1 \\
\hline Kansakrom Under Kukuom Service Center & 1 & 1 \\
\hline Kenyasi & 2 & 2 \\
\hline Kofiko (Drobo) & 1 & 1 \\
\hline Kukuom & 3 & 3 \\
\hline Kwabena Tenten & 1 & 1 \\
\hline Mim & 10 & 11 \\
\hline Mim Sakyikrom & 1 & 1 \\
\hline Nkrankwanta & 1 & 1 \\
\hline Nsapor (Berekum) & 1 & 1 \\
\hline Ntotroso/Kenyasi & 1 & 1 \\
\hline Sampa & 2 & 2 \\
\hline Sampa Atamfoso & 1 & 1 \\
\hline Sampa Buko & 1 & 1 \\
\hline Sampa (Small Water) & 1 & 1 \\
\hline Sankore (Kukuom) & 1 & 1 \\
\hline Sunyani & 12 & 13 \\
\hline Tepa & 8 & 9 \\
\hline Wanfie & 3 & 3 \\
\hline
\end{tabular}

Table 4: Model-error metrics and computational time

\begin{tabular}{lr}
\hline RMSE & 0.001639 \\
\hline MAE & 0.001321 \\
R & 0.034323 \\
Training time & 0.392 secs \\
Testing Time & 0.013 secs \\
\hline
\end{tabular}




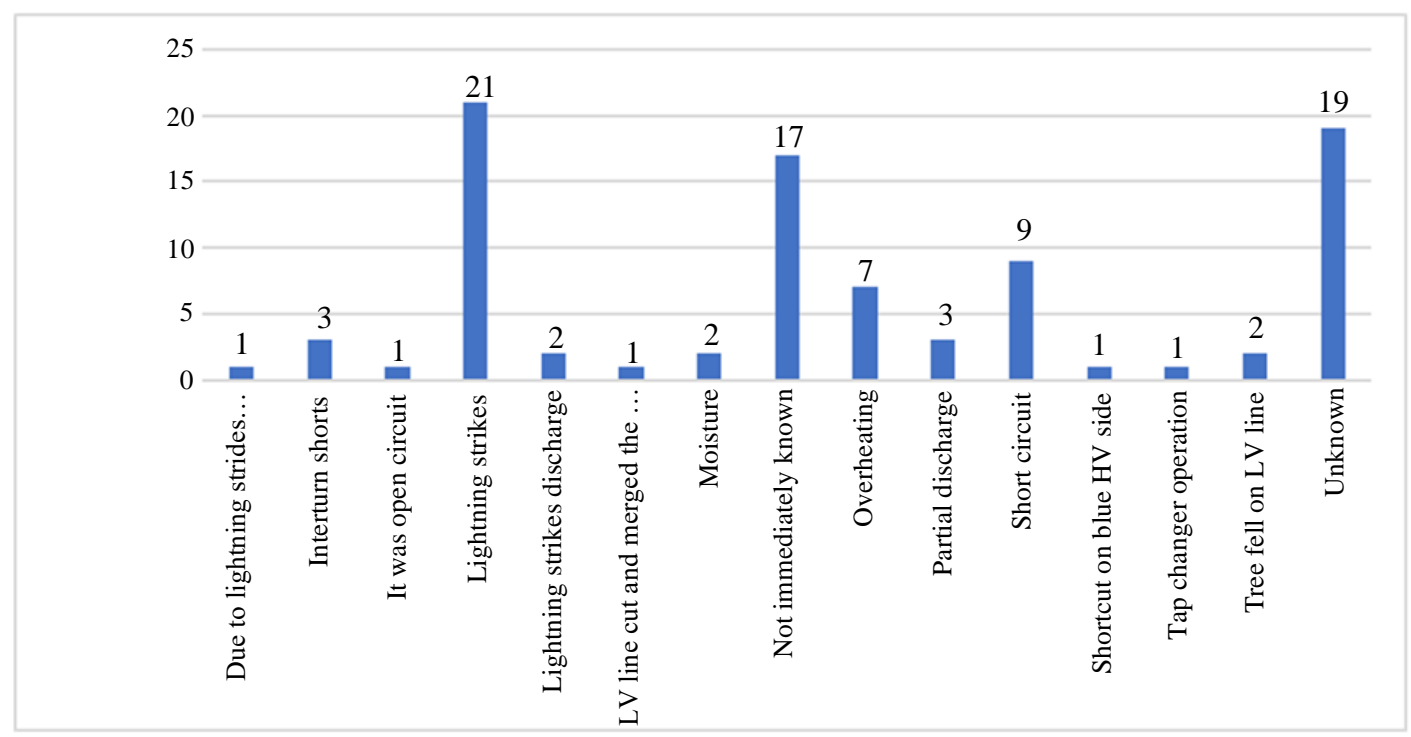

Fig. 2: Causes of damage

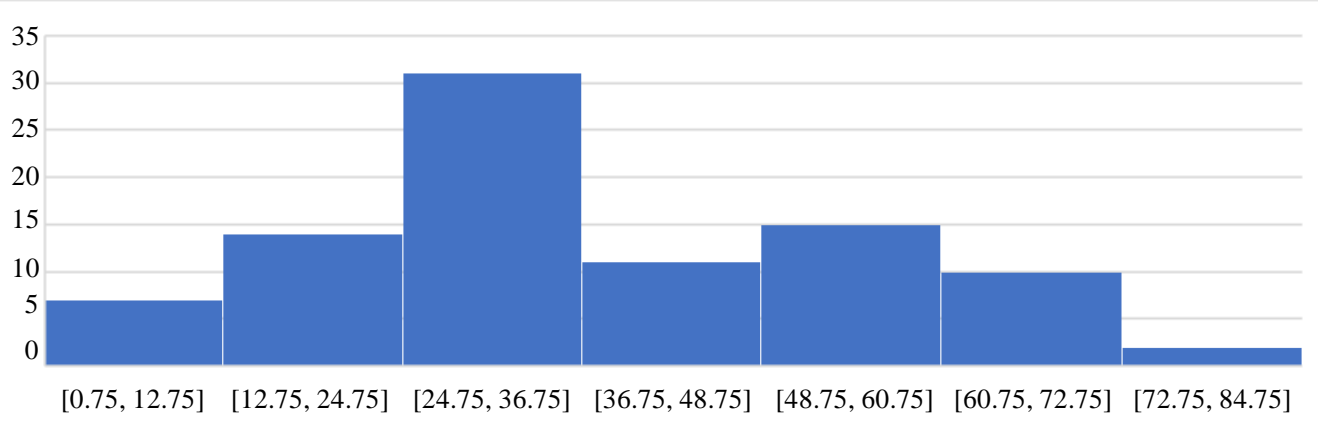

Fig. 3: Percentage age of the transformer before damage

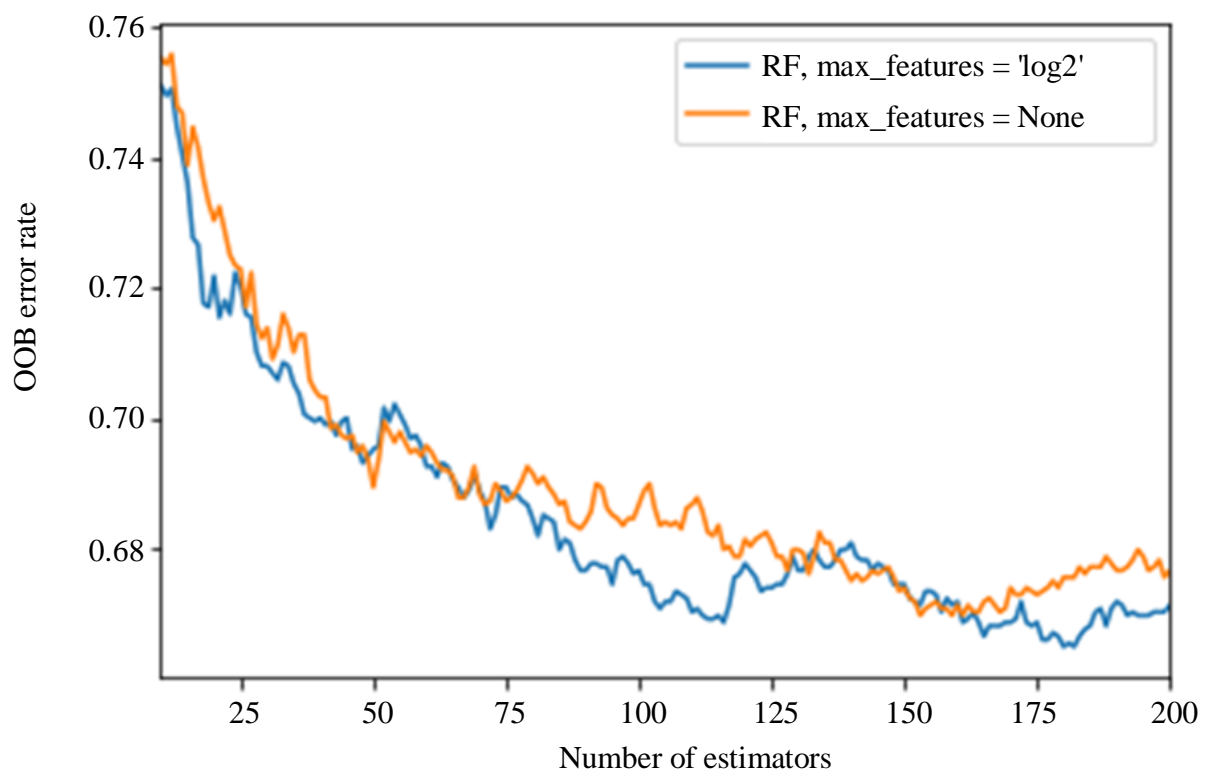

Fig. 4: Out-of-bag training error 


\section{Accuracy and Error Metrics}

Table 4 shows the error metrics and computational time of the proposed predictive model. From the feature ranking, the best eight (8) features (Fig. 5) were selected. These include transformer-rating, make, tap-changertype, voltage-level, output-voltage, output-current, typeof-faults sustained by a transformer and rate-of-faultyoccurrence as the input feature (independent variables) to predict the expected life in service $(y)$. The date set was partition into $(75 \%)$ training and $(25 \%)$ testing.

With a training dataset of seven hundred and fifty (750) rows eleven (9) columns, it took the proposed model $0.392 \mathrm{sec}$ to study all hidden pattern in the dataset. While 0.013 was taken to make predictions on two hundred and fifty (250) dataset. The RMSE of 0.001639 and MAPE error of 0.001321 achieved by the proposed model shows that the proposed model fits very well to the dataset. Again, the obtained error values indicate a comparable predicted value by the model compared with the actual values.

Figure 6 shows a visual plot of the model predicted expected life against the actual values of eight transformers. The nearness of the predicted-values to the actual-values affirms the low error metrics recorded. Thus, the values show that the proposed predictive framework predicts the response accurately in general. The study results demonstrate that machine learning models are useful in estimating the remaining life of the transformer.

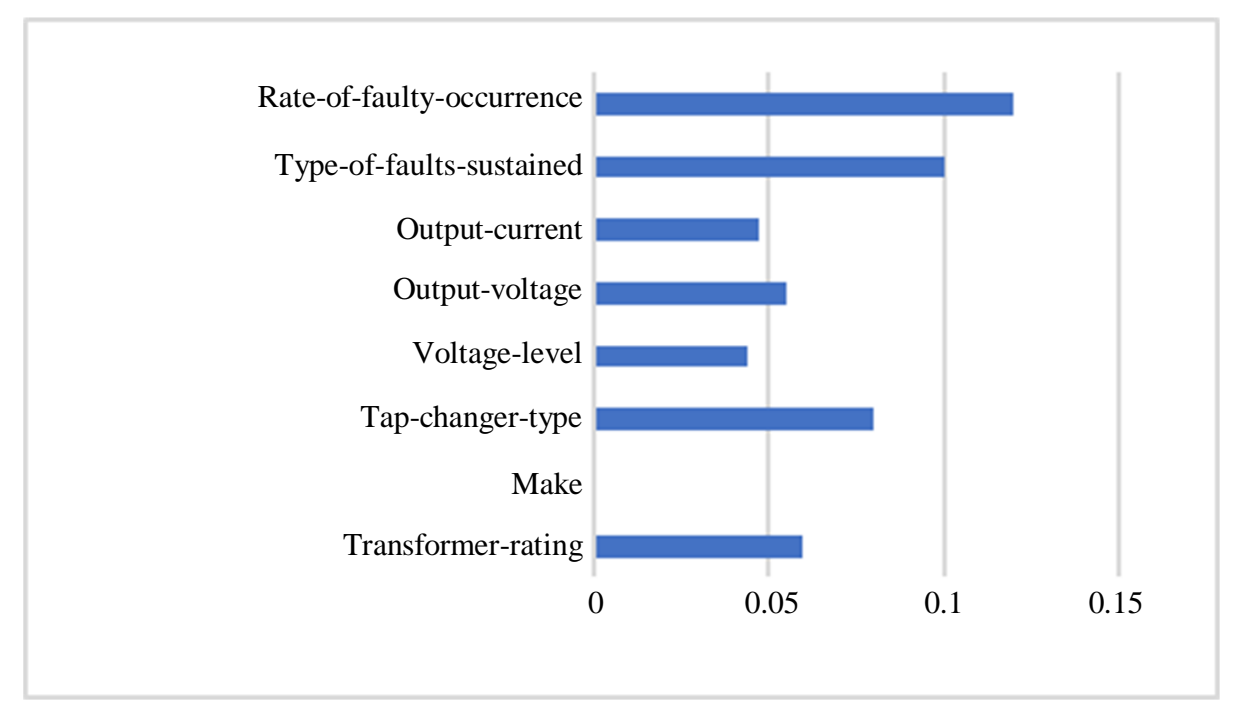

Fig. 5: Feature ranking of feature

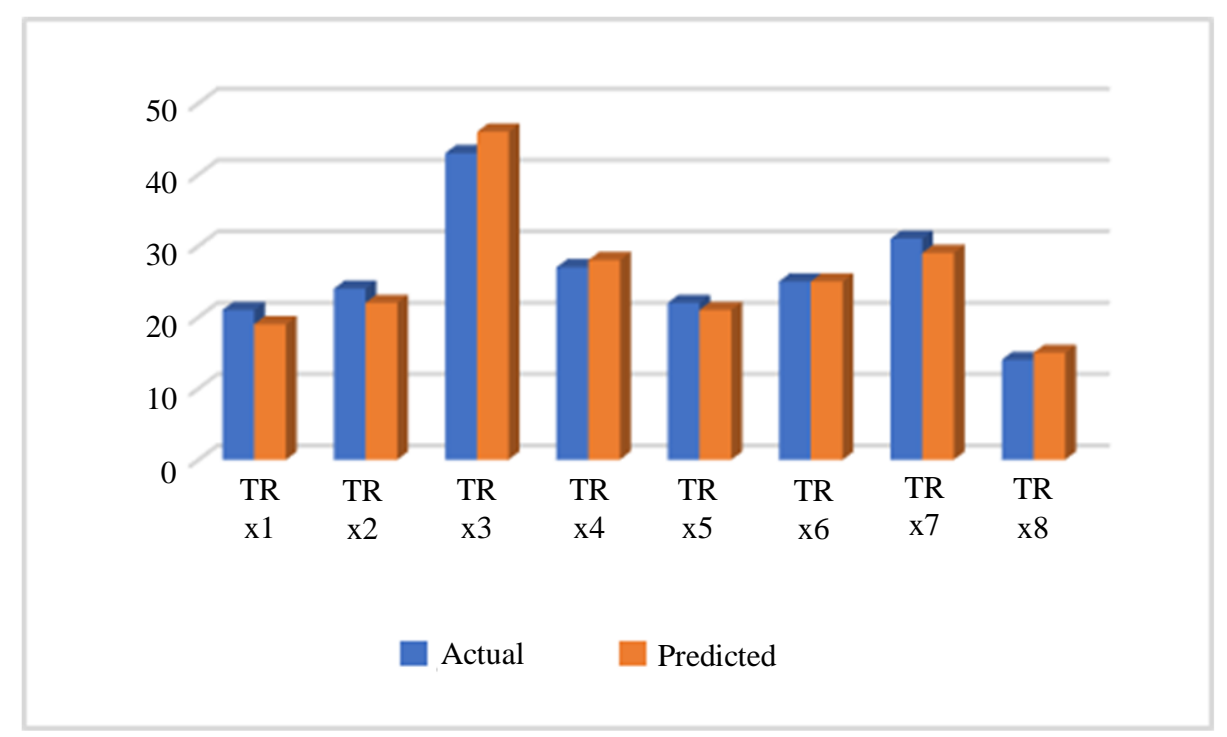

Fig. 6: A plot of model-predicted value vs actual values 


\section{Conclusion}

Transformers are a vital element in the power industry. Due to the impotence in the industry, they usually are designed to last for ample time, thus, at least 15 years. However, literature has shown that most transformers in service do not live to their expected life. Hence, several studies have been undertaken in the past to examine the causes of their failures. Also, estimate the remaining life of transformers before failure. However, most of these studies were concentrated in developed countries. Thus, little or no have been done in developing countries such as Ghana. Nevertheless, most of these studies reported that most factors that affect the unexpected failures of transformers are due to usage characters and environmental factors such as weather and temperature. However, these parameters differ from region to region.

In this study, we sought to examine the factors that contribute to the damage of distribution transformers in three regions of Ghana. Also, predict the remaining life of a distribution transformer in services based on the identified factors using machine learning. The accuracy of the proposed model was assessed through historical daily monitoring data of different distribution transformer of dissimilar rating, age-ofused and pre-known health conditions and operatingconditions from August 2010 to June 2019. The proposed model is anticipated to offer a faster approach for estimating the remaining life of transformers and assist novice engineers in this field to propose right risk-informed lifetime management decision based on current transformer conditions. The following conclusion was made:

1. A total of 90 distribution transformer were damaged within nine years. Thus, average the company losses ten (10) transformer in a year, which amount to the US \$92300-95770 per year

2. Most of the places that recorded rate of distribution transformer damage were the location that had mini and major factories around. Thus, the Sunyani Municipality recorded the highest transformer damage (12), representing 13\%, followed by Mim (10)

3. Lighting strike was the significant causes of transformer damage. Thus twenty-one (21) out of the ninety (90) damage transformers was caused by a lightning strike

4. The results show that $33.33 \%$ of the damage transformers were with $24.75-36.75 \%$ of their life expectancy. As low as $3.33 \%$ of the damage transformers have been in service for $73 \%$ of the life expectancy. From the study results, it can be concluded that a high percentage $(68.9 \%)$ of the damage transformers in the Bono, Bono East and Ahafo regions of Ghana have been in service less the half of its expected years of service

5. The result shows that the Rate-of-faulty-occurrence, Type-of-faults-sustained and Tap-changer-type are the most significant factors that determine the number of years left for a distribution transformer to fail. It was observed that the make of the transformer is of less importance in predicting the years left for a transformer to fail

The RMSE of 0.001639 and MAPE error of 0.001321 achieved by the proposed model shows that the proposed model fits very well to the dataset. From the fallout of this work, the following recommendations are made:

1. Looking at the high rate of transformer damage due to a lightning strike, we recommend that the authorities put in place correct and valid lighting arrestors to curb the rate of damage

2. The authorities of electricity distribution in the Sunyani municipality can implement this predictive model to help ascertain the remaining life of distribution transformers currently in service

3. We recommend authorities to intensify the maintenance schedules to reduce the failure rate

Notwithstanding, the accuracy obtained in the current study, data scarcity was a challenge, which, in a way, limited the accuracy of the current study. However, we believe that transfer learning techniques using the deep neural network can be adopted in future studies to overcome the limitation posed by the data size and improve prediction accuracy.

\section{Acknowledgement}

We wish to express our gratitude to all lecturers at the department of computer science and Electrical \& Electronic. To our faculty guide we say thank you very much.

\section{Funding Information}

The authors declare that they did not receive any funding for the current study.

\section{Author Contributions}

Justice Aning: Conceptualization, methodology, software, writing - original draft, data curation: Review, editing and revision.

Puguo Gbene George: Revised methodology, review, editing and revision.

Ntiminity Abontakoyah Enoch: Review, editing and revision. 


\section{Conflict of Interest}

The authors declare that they have no conflict of interest

\section{Reference}

Adejo, O. W., \& Connolly, T. (2018). Predicting student academic performance using multi-model heterogeneous ensemble approach. Journal of Applied Research in Higher Education.

Airlangga, G., \& Liu, A. (2019, March). Initial Machine Learning Framework Development of Agriculture Cyber Physical Systems. In Journal of Physics: Conference Series (Vol. 1196, No. 1, p. 012065). IOP Publishing.

Alibaba.com. (2018). Cost of distribution transformers. www.alibaba.com/showroom/power-distributiontransformer

Alvarez, D. L., Rivera, S. R., \& Mombello, E. E. (2019). Transformer Thermal Capacity Estimation and Prediction Using Dynamic Rating Monitoring. IEEE Transactions on Power Delivery, 34(4), 1695-1705.

Badune, J., Vītoliņa, S., \& Maskaḷonoks, V. (2013). Methods for predicting remaining service life of power transformers and their components.

Berhanu, F., \& Abera, A. (2015). Students' Performance Prediction based on their Academic Record. International Journal of Computer Applications, 131(5), 0975-8887.

Dai, Y., \& Zhang, Y. (2013). Machine learning in stock price trend forecasting.

Dewangan, T., \& Patel, P. (2017). Prediction of Distribution Transformer Premature Failures. International Journal for Research in Applied Science \& Engineering Technology (IJRASET), 5(V), 1450-1455.

Di, X. (2014). Stock trend prediction with technical indicators using SVM. Independent Work Report, Stanford Univ.

Faggella, D. (2018). What is machine learning? Techemergence. 2017.

Futoma, J., Simons, M., Panch, T., Doshi-Velez, F., \& Celi, L. A. (2020). The myth of generalisability in clinical research and machine learning in health care. The Lancet Digital Health, 2(9), e489-e492.

Gorgan, B., Notingher, P. V., Wetzer, J. M., Verhaart, H. F., Wouters, P. A., Van Schijndel, A., \& Tanasescu, G. (2012, May). Calculation of the remaining lifetime of power transformers paper insulation. In 2012 13th International conference on optimization of electrical and electronic equipment (OPTIM) (pp. 293-300). IEEE.

Hong, Y., Meeker, W. Q., \& McCalley, J. D. (2009). Prediction of remaining life of power transformers based on left truncated and right censored lifetime data. The Annals of Applied Statistics, 3(2), 857-879.
Hosseinkhanloo, M., Motlagh, S. H. M., Jahromi, A. N., \& Kalantari, N. T. (2020). Improving the ageing rate of transformer fleet using imperialist competitive algorithm. IET Generation, Transmission \& Distribution.

Huber, P., Gerber, M., Rumsch, A., \& Paice, A. (2018). Prediction of domestic appliances usage based on electrical consumption. Energy Informatics, 1(1), 16.

Kimment, M., \& Matevosyan, N. (2018). Predicting Distribution Transformer Failures.

Larivière, B., \& Van den Poel, D. (2005). Predicting customer retention and profitability by using random forests and regression forests techniques. Expert Systems with Applications, 29(2), 472-484.

Lin, J., Su, L., Yan, Y., Sheng, G., Xie, D., \& Jiang, X. (2018). Prediction method for power transformer running state based on LSTM_DBN network. Energies, 11(7), 1880.

Luo, M., \& Zhang, K. (2014). A hybrid approach combining extreme learning machine and sparse representation for image classification. Engineering Applications of Artificial Intelligence, 27, 228-235.

Martin, D., Cui, Y., Ekanayake, C., Ma, H., \& Saha, T. (2014). An updated model to determine the life remaining of transformer insulation. IEEE Transactions on power delivery, 30(1), 395-402.

Mharakurwa, E. T., Nyakoe, G. N., \& Akumu, A. O. (2019, June). Transformer Remnant Life Estimation and Asset Management model based on Insulation Stress Assessment. In 2019 IEEE Electrical Insulation Conference (EIC) (pp. 325-329). IEEE.

Ngiam, K. Y., \& Khor, W. (2019). Big data and machine learning algorithms for health-care delivery. The Lancet Oncology, 20(5), e262-e273.

Nti, I. K., \& Quarcoo, J. A. (2019). Self-motivation and academic performance in computer programming language using a hybridised machine learning technique. Int. J. Artif. Intell. Expert Syst, 8(2), 12-30.

Nti, I. K., Samuel, A. A., \& Michael, A. (2019a). Predicting Monthly Electricity Demand Using Soft-Computing.

Nti, K. O., Adekoya, A., \& Weyori, B. (2019b). Random forest based feature selection of macroeconomic variables for stock market prediction. American Journal of Applied Sciences, 16(7), 200-212.

Nti, I. K., Adekoya, A. F., \& Weyori, B. A. (2020a). A systematic review of fundamental and technical analysis of stock market predictions. Artificial Intelligence Review, 1-51.

Nti, I. K., Adekoya, A. F., \& Weyori, B. A. (2020b). Predicting Stock Market Price Movement Using Sentiment Analysis: Evidence from Ghana. Applied Computer Systems, 25(1), 33-42. 
Nti, I. K., Adekoya, A. F., \& Weyori, B. A. (2020c). Efficient Stock-Market Prediction Using Ensemble Support Vector Machine. Open Computer Science, 10(1), 153-163.

Nti, I. K., Adekoya, A. F., \& Weyori, B. A. (2020d). A comprehensive evaluation of ensemble learning for stock-market prediction. Journal of Big Data, 7(1), 1-40.

Nti, I. K., Appiah, A. Y., \& Nyarko-Boateng, O. (2020e). Assessment and prediction of earthing resistance in domestic installation. Engineering Reports, 2(1), e12090.

Nti, I. K., Teimeh, M., Adekoya, A. F., \& Nyarkoboateng, O. (2020f). Forecasting electricity consumption of residential users based on lifestyle data using artificial neural networks. ICTACT Journal on Soft Computing, 6956(April), 2107-2116.

Nti, I. K., Teimeh, M., Nyarko-Boateng, O., \& Adekoya, A. F. (2020g). Electricity load forecasting: A systematic review. Journal of Electrical Systems and Information Technology, 7(1), 1-19.

Osorio, C., \& Sawant, N. (2003). Transformer Lifetime Prediction.

http://www.stanford.edu/class/ee292k/reports/Christ ianNandan.pdf

Pickster, K. (2015). Determination of probability of failure of power transformers using statistical analysis (Doctoral dissertation).

Rawal, P., \& Pandya, V. (2015). Distribution Transformer Future Failure Prediction using Extreme value model. April, 68-72.
Said, D. M., Nor, K. M., \& Majid, M. S. (2010, September). Analysis of distribution transformer losses and life expectancy using measured harmonic data. In Proceedings of 14th International Conference on Harmonics and Quality of PowerICHQP 2010 (pp. 1-6). IEEE.

Tran, T. O., Dang, H. T., Dinh, V. T., \& Phan, X. H. (2017). Performance prediction for students: A multi-strategy approach. Cybernetics and Information Technologies, 17(2), 164-182.

Vaghela, C., Bhatt, N., \& Mistry, D. (2015). A survey on various classification techniques for clinical decision support system. International Journal of Computer Applications, 116(23).

Van Schijndel, A., Wetzer, J. M., \& Wouters, P. A. A. F. (2006, October). Forecasting transformer reliability. In 2006 IEEE Conference on Electrical Insulation and Dielectric Phenomena (pp. 577-582). IEEE.

Visser, K., \& Brihmohan, Y. (2008). The effectiveness of oil analysis for failure prediction of power transformers. Electricity Distribution Maintenance Summit.

Wiens, J., Saria, S., Sendak, M., Ghassemi, M., Liu, V. X., Doshi-Velez, F.,... \& Ossorio, P. N. (2019). Do no harm: A roadmap for responsible machine learning for health care. Nature medicine, 25(9), 1337-1340.

Wu, D., Jennings, C., Terpenny, J., Gao, R. X., \& Kumara, S. (2017). A comparative study on machine learning algorithms for smart manufacturing: Tool wear prediction using random forests. Journal of Manufacturing Science and Engineering, 139(7). 